\title{
The hyperbolic factor: A measure of time inconsistency
}

\author{
Kirsten I. M. Rohde
}

Published online: 14 July 2010

(C) The Author(s) 2010. This article is published with open access at Springerlink.com

\begin{abstract}
Many studies have found that discounting is hyperbolic rather than constant. Hyperbolic discounting induces time-inconsistent behavior and is becoming increasingly popular in economic applications. Most studies that provide evidence in favor of hyperbolic discounting either are merely qualitative or they depend on assumptions about, or parametric fittings of, utility functions. This paper provides a quantitative measure for the degree of deviation from stationarity and the induced time-inconsistency that can overcome the problems mentioned. This measure, the hyperbolic factor, also provides simple preference foundations of the most popular discount functions. Moreover, it can easily be calculated from data and does not require knowledge of utility. Thus, the hyperbolic factor provides an easy tool for theoretical preference foundations, critical empirical tests, and quantitative measurements of hyperbolic discounting.
\end{abstract}

Keywords Hyperbolic discounting • Hyperbolic factor • Time preference • Time-inconsistency

\section{JEL Classification D90}

Since Samuelson's (1937) introduction of constant discounted utility, this model has been widely accepted as a normative and descriptive model of intertemporal choice. According to general, possibly non-constant, discounted utility, a stream of outcomes is evaluated by first determining the utility of every

K. I. M. Rohde ( $ه)$

Erasmus School of Economics, H13-27, P. O. Box 1738,

3000 DR Rotterdam, The Netherlands

e-mail: rohde@ese.eur.nl 
outcome, i.e. the value of the outcome if it would be received immediately, then multiplying each utility by a discount factor that corresponds to the timepoint of receipt, and finally summing over these discounted utilities. Constant discounting implies that a preference between two streams of outcomes is not affected if all outcomes in both streams are delayed by a common amount of time.

In the last few decades, there has been an increasing number of empirical studies suggesting that discounting is not constant, including Benzion et al. (1989), Bleichrodt and Johannesson (2001), Cairns and van der Pol (2000), Green et al. (1994), Kirby and Marakovic (1995), Mazur (1987, 2001), Read and Read (2004), Rodriguez and Logue (1988), and Thaler (1981). If an early reward and another, later and larger reward are perceived as being equivalent, then delaying both rewards equally will, for most people, result in a strict preference for the later and larger reward, revealing decreasing impatience.

As a consequence of decreasing impatience, individuals' preferences can be time-inconsistent. Consider a person who prefers to receive two apples in one year plus one day rather than one apple in one year, but prefers to receive one apple today rather than two apples tomorrow (Thaler 1981). This person is decreasingly impatient. If his preferences between 'today' and 'tomorrow' remain the same for one year, and he resets the clock at zero whenever he makes a decision, then in one year from now he will prefer to receive one apple on that day rather than two apples one day later. Thus, his preferences between the two options will have changed over time. In this sense decreasing impatience may be viewed as reflecting an irrationality. Since Strotz's (1956) discussion of time-inconsistent preferences many economic models are adapted to incorporate changing preferences (e.g. Pollak 1968; Phelps and Pollak 1968; Peleg and Yaari 1973; Asheim 1997; Gul and Pesendorfer 2005; Eliaz and Spiegler 2006).

This paper proposes a simple and novel method to quantify the degree of deviation from stationarity. Remarkably, we do not need measurements of or assumptions about utilities of outcomes to determine the degree of timeinconsistency. That is, a measure of decreasing impatience is introduced, the hyperbolic factor, which can easily be calculated from data without knowledge of utility. One major advantage of the approach in this paper is that it can easily be applied to domains of outcomes that are hard to quantify, like, for instance, health states and environmental conditions. Since utilities and outcomes cancel out in our approach, outcomes can be taken to be anything.

The hyperbolic factor isolates the time inconsistent component of time preferences. Thereby, it provides a potentially interesting predictor for individual behavior in the field, like, for instance, smoking, exercising, dieting, and saving. One approach to construct a measure of decreasing impatience would be to find out how impatience changes over time. This would require knowledge of impatience at each time-point, i.e. knowledge of the entire discount function. Then, to determine this discount function, we would also need to know the utility function. It is, indeed, commonly believed in the field that such a 
procedure must be followed. Most studies implicitly assume that utility is linear (Chabris et al. 2008; Viscusi et al. 2008; Kinari et al. 2009).

Surprisingly, as this paper shows, we do not need to go through all these steps. In fact, measuring the degree of decreasing impatience is even easier than measuring the discount function. The hyperbolic factor can be obtained from only two indifferences. Suppose a decision maker is indifferent between receiving $\$ 105$ in $t$ weeks and receiving $\$ 100$ today. Suppose he is also indifferent between receiving $\$ 105$ in $t+\tau$ weeks and receiving $\$ 100$ in $\sigma$ weeks. Thus, if the receipt of $\$ 100$ is delayed by $\sigma$ weeks, the receipt of $\$ 105$ must be delayed by $\tau$ weeks in order to maintain indifference. The hyperbolic factor is equal to $(\tau-\sigma) /(t \sigma)$.

Increasingly popular models that capture decreasing impatience are hyperbolic and quasi-hyperbolic discounting (Phelps and Pollak 1968; Laibson 1997; Loewenstein and Prelec 1992; Harvey 1986, 1995; Mazur 1987). These models have been used in many fields (Akerlof 2002; Harris and Laibson 2001; Krusell and Smith 2003; Laibson 1997; Luttmer and Mariotti 2003; O'Donoghue and Rabin 1999; Rubinstein 2003, 2006; Thaler and Benartzi 2004). Most studies that provide empirical evidence in favor of (quasi-)hyperbolic discounting assume a particular, often linear, utility function or first need to parametrically fit utility. Thus, the quantitative evidence in favor of (quasi-)hyperbolic discounting is confounded by assumptions about and parametric fittings of utility. Most qualitative studies in favor of (quasi-)hyperbolic discounting only reject constant discounting and provide evidence in favor of general decreasing impatience, not of (quasi-)hyperbolic discounting in particular.

As will be shown, the hyperbolic factor is also a useful tool for testing the most popular discount functions in the literature. A constant positive hyperbolic factor corresponds to generalized hyperbolic discounting (Loewenstein and Prelec 1992). Quasi-hyperbolic discounting holds if and only if the hyperbolic factor is equal to zero for all points in time except the present (Phelps and Pollak 1968). If, in addition, the hyperbolic factor is zero at present, then constant discounting holds. Thus, estimating the hyperbolic factor and testing whether it is constant will be useful in testing which of the models fit empirical data best and in testing whether these models are appropriate at all or whether different models are called for. In contrast to what is done by almost every study in the literature on inter-temporal choice, this approach does not require assumptions about or estimations of utility.

The ideas in this paper are inspired by Prelec (2004), who introduced another measure of decreasing impatience and related it to investment behavior. Relative to the hyperbolic factor, his measure is harder to obtain from individuals in practice: it uses the second derivative of the logarithm of the discount function, which can only be obtained after measurements of discounting and utility. Moreover, the hyperbolic factor is model-free, i.e. it can also be used as a measure of decreasing impatience when preferences cannot be represented by discounted utility. This is not the case for Prelec's measure, which essentially needs a discount function. 
Section 1 defines the hyperbolic factor. This factor is applied to discounted utility in Section 2. All proofs are in the Appendix.

\section{The hyperbolic factor defined}

Let $\mathcal{X}=\mathbb{R}^{m}$ be a set of outcomes ${ }^{1}$ and $\mathcal{T}=\mathbb{R}_{+}$a set of time-points. A timed outcome $(t, \mu)$ yields outcome $\mu$ at time $t$ and nothing $(=0)$ at all other points in time, where $t=0$ corresponds to 'today.' We examine preferences $\succcurlyeq$ over timed outcomes. The relations $\preccurlyeq, \succ, \prec, \sim$ are as usual. Preferences over outcomes are derived from preferences over timed outcomes consumed today, i.e. $\chi \succcurlyeq \mu$ if and only if $(0, \chi) \succcurlyeq(0, \mu)$. We use $\mu$ as the greek analogue of ' $m$ ' to denote moderate outcomes and $\chi$ as a greek analogue (visually) of ' $x$ ' to denote extreme outcomes. Thus, $\chi$ will denote the larger gain or the larger, or more severe, loss.

We assume that $\succcurlyeq$ is a weak order, i.e $\succcurlyeq$ is complete $((s, \mu) \succcurlyeq(t, \chi)$ or $(t, \chi) \succcurlyeq$ $(s, \mu)$ for all $\mu, \chi \in \mathcal{X}$ and $s, t \in \mathcal{T}$, possibly both) and transitive. Preferences are monotonic if $\chi \succcurlyeq \mu$ implies $(t, \chi) \succcurlyeq(t, \mu)$ for every $t \in \mathcal{T}$, and $\chi \succ \mu$ implies $(t, \chi) \succ(t, \mu)$ for every $t \in \mathcal{T}$. Preferences are impatient if for every $s<t, \chi \succ 0$ implies $(s, \chi) \succ(t, \chi)$ and $\chi \prec 0$ implies $(s, \chi) \prec(t, \chi)$. Preferences are continuous if for every $(t, \chi)$ the sets $\{(s, \mu) \in \mathcal{T} \times \mathcal{X} \mid(s, \mu) \succcurlyeq(t, \chi)\}$ and $\{(s, \mu) \in \mathcal{T} \times \mathcal{X} \mid(s, \mu) \preccurlyeq(t, \chi)\}$ are closed. Throughout this paper we make the following assumption.

Assumption Preferences constitute a continuous, monotonic, and impatient weak order.

Consider two equivalent timed outcomes $(s, \mu) \sim(t, \chi)$, with $s<t$ and $\mu \nsim \chi$. Then we have either $\chi \succ \mu \succ 0$ or $\chi \prec \mu \prec 0$ ( $\mu$ is 'moderate' and $\chi$ is 'extreme'). If the outcome $\mu$ is delayed by time $\tau$, then stationarity implies that the outcome $\chi$ should also be delayed by $\tau$ in order to maintain indifference. Thus, under stationarity $(s, \mu) \sim(t, \chi)$ implies $(s+\tau, \mu) \sim(t+\tau, \chi)$. Stationarity reflects constant impatience.

The preference relation $\succcurlyeq$ exhibits decreasing impatience if for all $s<t$, $\tau \in \mathcal{T}$, (i) $\chi \succ \mu \succ 0$ and $(s, \mu) \sim(t, \chi)$ imply $(t+\tau, \chi) \succcurlyeq(s+\tau, \mu)$, and (ii) $\chi \prec \mu \prec 0$ and $(s, \mu) \sim(t, \chi)$ imply $(t+\tau, \chi) \preccurlyeq(s+\tau, \mu)$. Increasing impatience holds if the implied preferences are always the reverse. Thus, with decreasing impatience, when we consider two equivalent timed outcomes, then delaying both outcomes equally will result in less distinction between the timepoints, and, thus, more preference for the timed outcome with the preferred outcome. In this sense, decreasing impatience reflects that a time difference becomes decreasingly important as it lies farther in the future.

\footnotetext{
${ }^{1}$ All results in this paper remain valid if $\mathcal{X}$ is a connected topological space containing a reference outcome called 'nothing.' $\mathcal{X}$ can, for instance, be any convex subset of $\mathbb{R}^{m}$ containing zero, or a set of non-quantified health states.
} 
Assume another preference relation $\succcurlyeq^{*}$, which also is a continuous, monotonic, and impatient weak order. Preferences $\succcurlyeq^{*}$ exhibit more decreasing impatience than $\succcurlyeq$ if for all $s<t, \tau, \sigma \in \mathcal{T}(i) \chi^{*} \succ^{*} \mu^{*} \succ^{*} 0,(s, \mu) \sim(t, \chi)$, $(s+\sigma, \mu) \sim(t+\tau, \chi)$, and $\left(s, \mu^{*}\right) \sim^{*}\left(t, \chi^{*}\right)$ imply $\left(t+\tau, \chi^{*}\right) \succcurlyeq^{*}\left(s+\sigma, \mu^{*}\right)$, and (ii) $\chi^{*} \prec^{*} \mu^{*} \prec^{*} 0,(s, \mu) \sim(t, \chi),(s+\sigma, \mu) \sim(t+\tau, \chi)$, and $\left(s, \mu^{*}\right) \sim^{*}$ $\left(t, \chi^{*}\right)$ imply $\left(t+\tau, \chi^{*}\right) \preccurlyeq^{*}\left(s+\sigma, \mu^{*}\right)$ (Prelec 2004).

Consider again two equivalent timed outcomes $(s, \mu) \sim(t, \chi)$ with $s<t$. Assume that $(s+\sigma, \mu) \sim(t+\tau, \chi)$. Decreasing (increasing) impatience implies that $\tau-\sigma>0(\tau-\sigma<0)$. An obvious measure of decreasing impatience is, therefore, $\tau-\sigma$. This measure $\tau-\sigma$, however, will depend on $s, t, \sigma, \mu$, and $\chi$, and will be hard to compare across different outcomes and timepoints. The main purpose of this paper is to propose a transformation of this measure that is better suited as a measure of decreasing impatience, and that can be compared across different outcomes and time-points. This proposed measure, the hyperbolic factor, is defined next. It is just as easily observable from preferences as $\tau-\sigma$ itself. Unlike $\tau-\sigma$, however, it will be constant, i.e. independent of $s, t, \sigma, \mu$ and $\chi$, for all hyperbolic discounting models currently used in the literature, as we will see in Section 2.

Outcomes $\mu, \chi \in \mathcal{X}$ and time-points $s, t, \sigma, \tau \in \mathcal{T}$, with $s<t, \tau>0$, form an indifference pair if

$$
(s, \mu) \sim(t, \chi) \text { and }(s+\sigma, \mu) \sim(t+\tau, \chi) .
$$

In these indifferences, waiting $t$ instead of $s$ offsets the same monetary advantage as waiting $t+\tau$ instead of $s+\sigma$. Such a use of pairs of indifferences (or preferences) to compare tradeoffs across attributes has been widely used in decision under uncertainty. Examples include Abdellaoui (2002, p. 726, Definition 6), Blavatskyy (2006, p. 320, TO method), Bleichrodt and Miyamoto (2003, p. 183, tradeoff consistency), Bouyssou and Pirlot (2003, p. 685), Chateauneuf (1999, p. 25, C.S.T.P.), Karni (2003), Schmidt and Zank (2001, p. 486, EL-tradeoff consistency), Skiadas (1997, p. 257, Axiom A10), and Wakker and Deneffe (1996, p. 1134, TO method). Applications to interpersonal comparisons are in Ebert (2004, p. 421, independence) and Pinto (1997, p. 73, PTO-3), and applications to inter-temporal choice, the topic of this paper, include Prelec (1998, p. 503, compound invariance).

Definition 1 For every indifference pair as in Eq. 1 the hyperbolic factor is defined as

$$
\frac{\tau-\sigma}{t \sigma-s \tau}
$$

Thus, in order to obtain the hyperbolic factor, we only need an indifference pair. One recipe to obtain an indifference pair is as given in the following theorem. 
Theorem 2 An indifference pair can be constructed as follows.

Step I Take any $\chi \nsim 0$ and any $s, t, \tau$ with $s<t$, and $\tau>0$;

Step II Find $\mu$ such that $(s, \mu) \sim(t, \chi)$;

Step III Find $\sigma$ such that $(s+\sigma, \mu) \sim(t+\tau, \chi)$.

In the second step $\mu$ exists, but may not be unique: any $\mu^{\prime}$ with $\mu^{\prime} \sim \mu$ will yield the same $\sigma$ in the third step. In the third step $\sigma$ exists and is unique.

For general preferences, Steps II and III may not always be solvable. For instance, there may not exist $\mu$ and $\sigma$ that satisfy Eq. 1. Our assumptions about preferences, however, imply that such a case can never arise, so that a $\mu$ and $\sigma$ as described can always be found.

In Attema et al. (2010) we did an experiment where we used a slightly different procedure to obtain indifference pairs. We fixed outcomes $\mu, \chi$, and time point $s$. Then we elicited a $t$ such that $(s, \mu) \sim(t, \chi)$. Finally, we elicited a $\tau$ such that $(t, \mu) \sim(t+\tau, \chi)$, thereby letting $s+\sigma=t$. A drawback of the method in that experiment is that in theory it can be the case that $t$ or $\tau$ cannot be found. Nevertheless, in the experiment such a case did not occur.

Now we can define the function $H$ for every $\chi \nsim 0, s<t$, and $\tau>0$, as

$$
H(s, t, \chi, \tau)=\frac{\tau-\sigma}{t \sigma-s \tau},
$$

where $\sigma$ is such that together with a $\mu$ and the arguments of $\mathrm{H}$, it yields an indifference pair as in Eq..$^{2}$ The function $H$ gives the hyperbolic factors. In general, $H$ need not always be regular, i.e. $H$ is infinite if $t \sigma=s \tau$, and negative in spite of strongly decreasing impatience if $t \sigma<s \tau$. Yet, as we will see later, for the most popular discounted utility models in the literature, regularity holds, i.e. for every indifference pair as in Eq. 1 we have $t \sigma>s \tau$. Imposing regularity amounts to imposing an upper bound on the degree of decreasing impatience, as we will show next.

Consider an indifference pair with corresponding $s, t, \sigma, \tau$. We saw before that $\tau-\sigma$ can be viewed as a measure of decreasing impatience. From impatience we know that $s-t<\tau-\sigma<\tau$. For increasing impatience we have $\tau-\sigma<0$, and therefore also $t \sigma>s \tau$. Thus, regularity does not restrict the degree of increasing impatience. Now say that moderate decreasing impatience holds if $0<\tau-\sigma<\tau(t-s) / t$ and that strongly decreasing impatience holds if $\tau-\sigma \geq \tau(t-s) / t>0$. Then imposing regularity amounts to ruling out strongly decreasing impatience.

Let us now summarize the main properties of the hyperbolic factor. It can easily be shown that non-negative hyperbolic factors correspond to decreasing impatience. We will see in Section 2 that hyperbolic discounting induces nonnegative hyperbolic factors, and, thus, decreasing impatience.

\footnotetext{
${ }^{2}$ It is irrelevant which $\mu$ is chosen as long as it satisfies Eq. 1. Therefore, $\mu$ is not an argument of the function $H$.
} 
Theorem 3 Let regularity hold. Preferences $\succcurlyeq$ exhibit decreasing impatience if and only if $H \geq 0$. Preferences $\succcurlyeq$ exhibit increasing impatience if and only if $H \leq 0$.

Note that a similar result would hold for any monotonic transformation of $H$. The hyperbolic factor indeed serves as a measure of decreasing impatience, as shown in the next theorem. Thus, it properly captures Prelec's (2004) relative decreasing impatience. When we consider another preference relation $\succcurlyeq^{*}$, then it is assumed that $\succcurlyeq^{*}$ is a continuous, monotonic and impatient weak order, and that the corresponding hyperbolic factors are given by $H^{*}(s, t, \chi, \tau)$.

Theorem 4 Let regularity hold. Preferences $\succcurlyeq^{*}$ exhibit more decreasing impatience than $\succcurlyeq$ if and only if $H^{*}\left(s, t, \chi^{*}, \tau\right) \geq H(s, t, \chi, \tau)$ for all $s, t, \tau, \chi, \chi^{*}$.

Thus, we have shown that the hyperbolic factor is an appropriate modelfree measure of decreasing impatience that can easily be obtained from an indifference pair. To illustrate the measurement of the hyperbolic factor when discounted utility does not hold, consider the following example.

Example 5 Assume that preferences over timed outcomes are represented by $V(t, x)=e^{-r(x) t} x$, where $r(x)$ is a positive function. We will go through the three steps of Theorem 2 to obtain the hyperbolic factor.

Step I Fix $x, t, \tau$ with $t, \tau>0$ and $x>0$.

Step II Determine $y$ such that $(0, y) \sim(t, x)$. It follows that $y=e^{-r(x) t} x$.

Step III Determine $\sigma$ such that $(\sigma, y) \sim(t+\tau, x)$. It follows that

$$
e^{-r(y) \sigma} y=e^{-r(x)(t+\tau)} x .
$$

Substituting $y=e^{-r(x) t} x$ yields

$$
e^{-r(y) \sigma} e^{-r(x) t} x=e^{-r(x)(t+\tau)} x,
$$

which yields

$$
e^{-r(y) \sigma}=e^{-r(x) \tau} .
$$

Thus,

$$
\sigma=\tau \frac{r(x)}{r(y)} .
$$

The hyperbolic factor is then given by

$$
H=\frac{\tau-\sigma}{t \sigma-s \tau}=\frac{\tau\left(1-\frac{r(x)}{r(y)}\right)}{\tau t \frac{r(x)}{r(y)}}=\frac{1}{t}\left(\frac{r\left(e^{-r(x) t} x\right)}{r(x)}-1\right) .
$$


Note that if $r(\cdot)$ is a constant function, we have the common constant discounting. In that case the hyperbolic factor will be zero, as we will also show in the next section.

\section{The hyperbolic factor and discounted utility}

Preferences $\succcurlyeq$ can be represented by a function $V: \mathcal{T} \times \mathcal{X} \rightarrow \mathbb{R}$ if $V(t, \chi) \geq$ $V(s, \mu) \Longleftrightarrow(t, \chi) \succcurlyeq(s, \mu)$. Discounted utility holds if there exist a discount function $\varphi$ and a utility function $u$ such that $\succcurlyeq$ can be represented by

$$
D U(t, \mu)=\varphi(t) u(\mu),
$$

where $\varphi$ is continuous and strictly decreasing, $\varphi(0)=1, \varphi(t)>0$ for every $t$, and $u$ is continuous, $u(0)=0$, and there is an outcome $\chi \in \mathcal{X}$ with $u(\chi) \neq 0$. Fishburn and Rubinstein (1982) characterized discounted utility. ${ }^{3}$ In this section we will assume that discounted utility holds. This implies a continuous, monotonic and impatient weak order as in Section 1. We will not assume regularity, but instead derive it later from other assumptions. When combined with Fishburn and Rubinstein's (1982) preference foundation of discounted utility, this section provides preference foundations for all currently popular discount models.

Under discounted utility, the hyperbolic factor is independent of the outcomes, as the following theorem shows.

Theorem 6 Let discounted utility hold. Then $H(s, t, \chi, \tau)$ is independent of $\chi$.

Thus, from now on we will write $H(s, t, \tau)$ instead of $H(s, t, \chi, \tau)$. If we know the discount function $\varphi$, then the hyperbolic factors can be calculated by

$$
H(s, t, \tau)=\frac{s+\tau-\varphi^{-1}\left(\frac{\varphi(s)}{\varphi(t)} \varphi(t+\tau)\right)}{t \varphi^{-1}\left(\frac{\varphi(s)}{\varphi(t)} \varphi(t+\tau)\right)-s(t+\tau)},
$$

where $\varphi^{-1}$ is the inverse of $\varphi$, which exists under our assumptions.

Two decision-makers with different discount functions $\varphi$ and $\varphi^{*}$ that are related by a power transformation $\varphi^{*}(t)=[\varphi(t)]^{c}$ have equal hyperbolic factors, as stated in Observation 7. Thus, in order to measure deviations from stationarity, we do not even need to know how much people discount in an absolute sense. This observation underlies the possibility to analyze decreasing impatience without a need to measure the discount function or utility. Thus, the hyperbolic factor isolates the time inconsistent component of time preferences.

\footnotetext{
${ }^{3}$ Lancaster (1963) used a slightly different approach to model preferences over timed outcomes.
} 
Observation 7 Let discounted utility hold. Consider two discount functions $\varphi$ and $\varphi^{*}$ with corresponding $H$ and $H^{*}$. If there is a $c \in \mathbb{R}$ such that $\varphi^{*}(t)=$ $[\varphi(t)]^{c}$ then $H^{*}(s, t, \tau)=H(s, t, \tau)$ for every $s, t, \tau$.

\subsection{Constant discounting}

Constant discounting has been a traditional assumption in economics. Preferences satisfy constant discounting if there is a constant discount factor $\delta$ such that $\varphi(t)=\delta^{t}$ for every $t$. Constant discounting is equivalent to the hyperbolic factor always being zero.

Theorem 8 The following two statements are equivalent under discounted utility.

(i) Preferences $\succcurlyeq$ satisfy constant discounting.

(ii) $H(s, t, \tau)=0$ for all $s, t, \tau$.

2.2 Generalized hyperbolic discounting

Following up on the empirical studies that found violations of stationarity, Loewenstein and Prelec (1992) introduced the generalized hyperbolic discount function, which is defined by

$$
\varphi(t)=(1+h t)^{-r / h},
$$

with $h>0, r>0$. Generalized hyperbolic discounting is equivalent to the hyperbolic factor being a positive constant.

Theorem 9 The following two statements are equivalent under discounted utility for each $h>0$.

(i) Preferences $\succcurlyeq$ satisfy generalized hyperbolic discounting $\varphi(t)=(1+$ $h t)^{-r / h}$.

(ii) $H(s, t, \tau)=h$ for all $s, t, \tau$.

Mazur (1987) tested a necessary condition for hyperbolic discounting that also did not require knowledge of utility. He showed that for all $s, t$ with $(s, \mu) \sim(t, \chi), t$ should depend linearly on $s$ with a positive intercept whenever generalized hyperbolic discounting holds with $r=h$. It can be shown that his condition also holds when $r \neq h$. We provide a testable condition that is not only necessary, but also sufficient for hyperbolic discounting, as Theorem 9 shows. The proof is derived from Loewenstein's and Prelec's (1992) and alNowaihi's and Dhami's (2006) sufficiency condition.

Harvey (1986) proposed a discount function given by

$$
\varphi(t)=(1+t)^{-r}
$$


This Harvey discounting is equivalent to generalized hyperbolic discounting with a hyperbolic factor that equals one.

\subsection{Proportional discounting}

Mazur (1987) and Harvey (1995) proposed a discount function given by

$$
\varphi(t)=(1+h t)^{-1} .
$$

This proportional discounting is equivalent to generalized hyperbolic discounting with $r=h$.

It follows that the hyperbolic factor does not distinguish between the generalized hyperbolic discounting of Loewenstein and Prelec (1992) and the proportional discounting of Mazur (1987) and Harvey (1995). This is because the hyperbolic factor only restricts the parameter $h$ and not the parameter $r$ as we saw in Observation 7. Indeed, on our domain of timed outcomes the two models cannot be distinguished, because they differ only regarding the absolute level of discounting and not regarding the degree of decreasing impatience. This finding reflects once more that changes in impatience and the corresponding irrationalities can be investigated independently of the absolute level of discounting.

\subsection{Quasi-hyperbolic discounting}

Phelps and Pollak (1968) introduced quasi-hyperbolic discounting, as used by Laibson (1997) and many others. The quasi-hyperbolic discount function is given by

$$
\varphi(t)= \begin{cases}1 & \text { if } t=0 \\ \beta \delta^{t} & \text { if } t>0\end{cases}
$$

for some $\beta \leq 1$, and some $\delta>0$.

Quasi-hyperbolic discounting is equivalent to stationarity for all of the future except the present.

Theorem 10 The following two statements are equivalent under discounted utility.

(i) Preferences $\succcurlyeq$ satisfy quasi-hyperbolic discounting with parameters $\beta$ and $\delta$.

(ii) $\quad H(s, t, \tau)=0$ for all $s>0, t, \tau$, and $H(0, t, \tau)=\frac{\ln (\beta)}{t(\tau \ln (\delta)-\ln (\beta))}$ for all $t, \tau$.

Contrary to what is commonly assumed, the degree of decreasing impatience in the quasi-hyperbolic model does not only depend on $\beta$, but also on $\delta$. Thus, it is $\beta$ in relation to $\delta$ that determines the degree of decreasing impatience. A similar observation was made by Prelec (2004). 


\section{Conclusion}

This paper has introduced the hyperbolic factor, a quantitative measure of decreasing impatience and time-inconsistency, which can easily be obtained from an indifference pair. In addition to being a simple measure, the hyperbolic factor is useful in characterizing all popular discount models. Generalized hyperbolic discounting holds if and only if the hyperbolic factor is constant and positive. Quasi-hyperbolic discounting holds if and only if the hyperbolic factor is equal to zero for all future points in time except the present. If, in addition, the hyperbolic factor is equal to zero today, then constant discounting holds.

This paper showed the value of obtaining indifference by searching for time points rather than outcomes. Many studies in the literature on inter-temporal choice elicit indifferences by varying outcomes, not by varying time points. The advantage of varying time points is that utilities can then cancel out, like in Casari (2009) and Onay and Öncüler (2007). Thus, by varying time points one can avoid assumptions about and estimations of utility, when testing the hypotheses that are often tested in the literature.

Acknowledgements The author would like to thank Han Bleichrodt and Drazen Prelec for helpful comments. Special thanks go to Peter P. Wakker from whom this work has benefited a lot.

Open Access This article is distributed under the terms of the Creative Commons Attribution Noncommercial License which permits any noncommercial use, distribution, and reproduction in any medium, provided the original author(s) and source are credited.

\section{Appendix}

Proof of Theorem 2 Consider $s, t, \chi, \tau$ with $0 \leq s<t, \chi \nsim 0$, and $\tau>0$. Assume that $\chi \succ 0$. By monotonicity and impatience we know that ${ }^{4}$

$$
(s, 0) \sim(t, 0) \prec(t, \chi) \prec(s, \chi) .
$$

By continuity of preferences and connectedness of $\mathbb{R}^{m}$ there must then be a $\mu$ with $(s, \mu) \sim(t, \chi)$ and $\chi \succ \mu \succ 0$. Thus,

$$
(s, \mu) \sim(t, \chi) \succ(t+\tau, \chi) \succ(t+\tau, \mu) .
$$

By continuity there must then be a $\sigma$ with $(s+\sigma, \mu) \sim(t+\tau, \chi)$. By replacing all ' $\succ$ ' by ' $\prec$ ' and all ' $\prec$ ' by ' $\succ$ ', this reasoning shows that similar things hold for $\chi \prec 0$.

By monotonicity and impatience, $\mu$ is unique up to indifference and $\sigma$ is unique.

\footnotetext{
${ }^{4} \mathrm{By}$ the definition of a timed outcome it follows that $(s, 0) \sim(t, 0)$ for every $s, t$.
} 
Proof of Theorem 3 By regularity, we have $H \geq 0$ if and only if $\tau-\sigma \geq 0$ for all indifference pairs as in Eq. 1. Thus, by Theorem 2, we have $H \geq 0$ if and only if decreasing impatience holds.

Proof of Theorem 4 By regularity, we have $H^{*}\left(s, t, \chi^{*}, \tau\right) \geq H(s, t, \chi, \tau)$ for every $s, t, \tau, \chi, \chi^{*}$ if and only if $\sigma^{*} \leq \sigma$ for all $s<t, \chi \nsim 0, \tau>0$ with $(s, \mu) \sim$ $(t, \chi),(s+\sigma, \mu) \sim(t+\tau, \chi)$ and $\left(s, \mu^{*}\right) \sim^{*}\left(t, \chi^{*}\right),\left(s+\sigma^{*}, \mu^{*}\right) \sim^{*}\left(t+\tau, \chi^{*}\right)$, which, by impatience, holds if and only if $\succcurlyeq^{*}$ exhibits more decreasing impatience than $\succcurlyeq$.

Proof of Theorem 6 Let $H(s, t, \chi, \tau)=h$ and $H\left(s, t, \chi^{*}, \tau\right)=h^{*}$. Then there are $\mu, \sigma, \mu^{*}, \sigma^{*}$, with $(s, \mu) \sim(t, \chi),(s+\sigma, \mu) \sim(t+\tau, \chi),\left(s, \mu^{*}\right) \sim\left(t, \chi^{*}\right)$ and $\left(s+\sigma^{*}, \mu^{*}\right) \sim\left(t+\tau, \chi^{*}\right)$. By discounted utility it follows that

$$
\varphi(s) u(\mu)=\varphi(t) u(\chi)
$$

and

$$
\varphi(s+\sigma) u(\mu)=\varphi(t+\tau) u(\chi) .
$$

Therefore,

$$
\frac{u(\mu)}{u(\chi)}=\frac{\varphi(t)}{\varphi(s)}=\frac{\varphi(t+\tau)}{\varphi(s+\sigma)} .
$$

Similarly,

$$
\frac{u\left(\mu^{*}\right)}{u\left(\chi^{*}\right)}=\frac{\varphi(t)}{\varphi(s)}=\frac{\varphi(t+\tau)}{\varphi\left(s+\sigma^{*}\right)} .
$$

By impatience it then follows that $\sigma^{*}=\sigma$ and $h=h^{*}$. This proves our result.

A similar reasoning proves Observation 7.

Proof of Theorem 8 Let $H(s, t, \chi, \tau)=0$ for all $s, t, \chi, \tau$. Then

$$
(s, \mu) \sim(t, \chi)
$$

if and only if

$$
(s+\tau, \mu) \sim(t+\tau, \chi),
$$

i.e. stationarity holds. Thus, for every $s, t, \sigma \in \mathcal{T}$,

$$
\frac{\varphi(s)}{\varphi(t)}=\frac{\varphi(s+\tau)}{\varphi(t+\tau)} .
$$

Therefore, by setting $s=0$, for every $t, \tau \in \mathcal{T}$,

$$
\varphi(t) \varphi(\tau)=\varphi(t+\tau) .
$$


By Cauchy's functional equation it follows that there must be a $c \in \mathbb{R}$ such that $\varphi(t)=e^{c t}$ for every $t \in \mathcal{T}$. Now let $\delta=e^{c}$. Then, $\varphi(t)=\delta^{t}$. The converse follows easily.

Proof of Theorem 9 Let there be a constant $h>0$ such that $H(s, t, \chi, \tau)=h$ for all $s, t, \chi, \tau$. Assume that

$$
u(\mu)=\varphi(t) u(\chi) \text { and } \varphi(\sigma) u(\mu)=\varphi(t+\tau) u(\chi)
$$

with $t>0$. Then we must have

$$
\frac{\tau-\sigma}{t \sigma}=h
$$

Let $k=1+h t$. It follows that $t+\tau=t+k \sigma$. Moreover, $k$ is a constant that depends only on $t$. Al-Nowaihi and Dhami (2006), inspired by Loewenstein and Prelec (1992), showed that this implies that the discount function is of the generalized hyperbolic form. Thus, there must be parameters $\tilde{h}, r$ such that $\varphi(t)=(1+\tilde{h} t)^{-r / \tilde{h}}$. It follows that $\tilde{h}=h$. From the assumption that $\varphi(\cdot)$ is strictly decreasing it follows that $r>0$. This proves one direction of our result. The proof of the other direction is straightforward.

Proof of Theorem 10 Let $H(s, t, \chi, \tau)=0$ for all $s>0, t, \chi, \tau$. Then for every $s, t, \sigma \in \mathcal{T}$, with $s, t>0$

$$
\frac{\varphi(s)}{\varphi(t)}=\frac{\varphi(s+\sigma)}{\varphi(t+\sigma)}
$$

Let $s_{0}>0$. Define the function $\varphi_{0}$ on $\mathcal{T}$ by $\varphi_{0}(t)=\varphi\left(t+s_{0}\right) / \varphi\left(s_{0}\right)$. Then for every $s, t, \sigma \in \mathcal{T}$,

$$
\frac{\varphi_{0}(s)}{\varphi_{0}(t)}=\frac{\varphi\left(s+s_{0}\right)}{\varphi\left(t+s_{0}\right)}=\frac{\varphi\left(s+s_{0}+\sigma\right)}{\varphi\left(t+s_{0}+\sigma\right)}=\frac{\varphi_{0}(s+\sigma)}{\varphi_{0}(t+\sigma)} .
$$

Moreover, $\varphi_{0}(0)=1$. By Cauchy's functional equation it follows that there must be a $c_{0} \in \mathbb{R}$ such that $\varphi_{0}(t)=e^{c_{0} t}$ for every $t \in \mathcal{T}$. Therefore, $\varphi\left(t+s_{0}\right)=$ $e^{c_{0} t} \varphi\left(s_{0}\right)$ for every $t \in \mathcal{T}$. Thus, $\varphi(t)=e^{c_{0}\left(t-s_{0}\right)} \varphi\left(s_{0}\right)$ for all $t \geq s_{0}$. Define $\delta_{0}=e^{c_{0}}$ and $\beta_{0}=e^{-c_{0} s_{0}} \varphi\left(s_{0}\right)$. Then $\varphi(t)=\beta_{0} \delta_{0}^{t}$ for all $t \geq s_{0}$. Similarly, consider an $s_{1}$ with $0<s_{1}<s_{0}$ and with corresponding $\beta_{1}$ and $\varphi_{1}$. It follows that for all $t \geq s_{0}$, $\varphi(t)=\beta_{1} \delta_{1}^{t}=\beta_{0} \delta_{0}^{t}$, so $\beta_{0}=\beta_{1}$ and $\delta_{0}=\delta_{1}$. We can continue this argument repeatedly.

Thus, letting $\beta=\beta_{0}$ and $\delta=\delta_{0}$ we obtain $\varphi(t)=\beta \delta^{t}$ for all $t>0$. By definition we have $\varphi(0)=1$.

Now, by letting $s=0$ we obtain $H(0, t, \tau)=\frac{\ln (\beta)}{t(\tau \ln (\delta)-\ln (\beta))}$ as follows. For an indifference pair

$$
(0, \mu) \sim(t, \chi) \text { and }(\sigma, \mu) \sim(t+\tau, \chi) .
$$


we have

$$
\frac{1}{\beta \delta^{t}}=\frac{\varphi(0)}{\varphi(t)}=\frac{\varphi(\sigma)}{\varphi(t+\tau)}=\frac{\beta \delta^{\sigma}}{\beta \delta^{t+\tau}}
$$

It follows that $1 / \beta=\delta^{(\sigma-\tau)}$. Thus, $\tau-\sigma=\ln (\beta) / \ln (\delta)$. The result follows.

\section{References}

Abdellaoui, M. (2002). A genuine rank-dependent generalization of the von NeumannMorgenstern expected utility theorem. Econometrica, 70, 717-736.

Akerlof, G. A. (2002). Behavioral macroeconomics and macroeconomic behavior. American Economic Review, 92, 411-433.

al-Nowaihi, A., \& Dhami, S. (2006). A note on the Loewenstein-Prelec theory of intertemporal choice. Mathematical Social Sciences, 52, 99-108.

Asheim, G. B. (1997). Individual and collective time-consistency. Review of Economic Studies, 64, 427-443.

Attema, A. E., Bleichrodt, H., Rohde, K. I. M., \& Wakker, P. P. (2010). Time-tradeoff sequences for analyzing discounting and time inconsistency. Management Science (forthcoming).

Benzion, U., Rapoport, A., \& Yagil, J. (1989). Discount rates inferred from decisions: An experimental study. Management Science, 35, 270-284.

Blavatskyy, P. R. (2006). Error propagation in the elicitation of utility and probability weighting functions. Theory and Decision, 60, 315-334.

Bleichrodt, H., \& Johannesson, M. (2001). Time preference for health: A test of stationarity versus decreasing timing aversion. Journal of Mathematical Psychology, 45, 265-282.

Bleichrodt, H., \& Miyamoto, J. (2003). A characterization of quality-adjusted life-years under cumulative prospect theory. Mathematics of Operations Research, 28, 181-193.

Bouyssou, D., \& Pirlot, M. (2003). Nontransitive decomposable conjoint measurement. Journal of Mathematical Psychology, 46, 677-703.

Casari, M. (2009). Pre-commitment and flexibility in a time decision experiment. Journal of Risk and Uncertainty, 38, 117-141.

Cairns, J., \& van der Pol, M. (2000). Valuing future private and social benefits: The discounted utility model versus hyperbolic discounting models. Journal of Economic Psychology, 21, 191-205.

Chabris, C. F., Laibson, D., Morris, C. L., Schuldt, J. P., \& Taubinsky, D. (2008). Individual laboratory-measured discount rates predict field behavior. Journal of Risk and Uncertainty, 37, 237-269.

Chateauneuf, A. (1999). Comonotonicity axioms and rank-dependent expected utility theory for arbitrary consequences. Journal of Mathematical Economics, 32, 21-45.

Ebert, U. (2004). Social welfare, inequality, and poverty when needs differ. Social Choice and Welfare, 23, 415-448.

Eliaz, K., \& Spiegler, R. (2006). Contracting with diversely naive agents. Review of Economic Studies, 73, 689-714.

Fishburn, P. C., \& Rubinstein, A. (1982). Time preference. International Economic Review, 23, 677-694.

Green, L., Fristoe, N., \& Myerson, J. (1994). Temporal discounting and preference reversals in choice between delayed outcomes. Psychonomic Bulletin and Review, 1, 383-389.

Gul, F., \& Pesendorfer, W. (2005). The revealed preference theory of changing tastes. Review of Economic Studies, 72, 429-448.

Harris, C., \& Laibson, D. (2001). Dynamic choices of hyperbolic consumers. Econometrica, 69, 935-957.

Harvey, C. M. (1995). Proportional discounting of future costs and benefits. Mathematics of Operations Research, 20, 381-399. 
Harvey, C. M. (1986). Value functions for infinite-period planning. Management Science, 32, $1123-1139$.

Karni, E. (2003). On the representation of beliefs by probabilities. Journal of Risk and Uncertainty, 26, 17-38.

Kinari, Y., Ohtake, F., \& Tsutsui, Y. (2009). Time discounting: Declining impatience and interval effect. Journal of Risk and Uncertainty, 39, 87-112.

Kirby, K. N., \& Marakovic, N. N. (1995). Modeling myopic decisions: Evidence for hyperbolic delay-discounting within subjects and amounts. Organizational Behavior and Human Decision Processes, 64, 22-30.

Krusell, P., \& Smith, A. A. (2003). Consumption-savings decisions with quasi-geometric discounting. Econometrica, 71, 365-375.

Laibson, D. (1997). Golden eggs and hyperbolic discounting. Quarterly Journal of Economics, 112, 443-477.

Lancaster, K. (1963). An axiomatic theory of consumer time preference. International Economic Review, 4, 221-231.

Loewenstein, G., \& Prelec, D. (1992). Anomalies in intertemporal choice: Evidence and an interpretation. Quarterly Journal of Economics, 107, 573-597.

Luttmer, E. G. J., \& Mariotti, T. (2003). Subjective discounting in an exchange economy. Journal of Political Economy, 111, 1-30.

Mazur, J. E. (1987). An adjusting procedure for studying delayed reinforcement. In J. E. Mazur, M. L. Commons, J. A. Nevin, \& H. Rachlin (Eds.), Quantitative analyses of behavior: The effect of delay and of intervening events on reinforcement value (Vol. 5, 55-73). Hillsdale, NJ: Erlbaum.

Mazur, J. E. (2001). Hyperbolic value addition and general models of animal choice. Psychological Review, 108, 96-112.

O'Donoghue, T., \& Rabin, M. (1999). Doing it now or later. American Economic Review, 89, $103-124$.

Onay, S., \& Öncüler, A. (2007). Intertemporal choice under timing risk: An experimental approach. Journal of Risk and Uncertainty, 34, 99-121.

Peleg, B., \& Yaari, M. E. (1973). On the existence of a consistent course of action when tastes are changing. Review of Economic Studies, 40, 391-401.

Phelps, E. S., \& Pollak, R. A. (1968). On second-best national saving and game-equilibrium growth. Review of Economic Studies, 35, 185-199.

Pinto, J. L. (1997). Is the person trade-off a valid method for allocating health care resources? Health Economics, 6, 71-81.

Pollak, R. A. (1968). Consistent planning. Review of Economic Studies, 35, 201-208.

Prelec, D. (1998). The probability weighting function. Econometrica, 66, 497-527.

Prelec, D. (2004). Decreasing impatience: A criterion for non-stationary time preference and 'hyperbolic' discounting. Scandinavian Journal of Economics, 106, 511-532.

Read, D., \& Read, N. L. (2004). Time discounting over the lifespan. Organizational Behavior and Human Decision Processes, 94, 22-32.

Rodriguez, M. L., \& Logue, A. W. (1988). Adjusting delay to reinforcement: Comparing choice in pigeons and humans. Journal of Experimental Psychology: Animal Behavior Processes, 14, 105-117.

Rubinstein, A. (2003). 'Economics and psychology'? The case of hyperbolic discounting. International Economic Review, 44, 1207-1216.

Rubinstein, A. (2006). Dilemmas of an economic theorist. Econometrica, 74, 865-883.

Samuelson, P. A. (1937). A note on measurement of utility. The Review of Economic Studies, 4, $155-161$.

Schmidt, U., \& Zank, H. (2001). A new axiomatization of rank-dependent expected utility with tradeoff consistency for equally likely outcomes. Journal of Mathematical Economics, 35, 483-491.

Skiadas, C. (1997). Subjective probability under additive aggregation of conditional preferences. Journal of Economic Theory, 76, 242-271.

Strotz, R. H. (1956). Myopia and inconsistency in dynamic utility maximization. Review of Economic Studies, 23, 165-180.

Thaler, R. H. (1981). Some empirical evidence of dynamic inconsistency. Economics Letters, 8, 201-207. 
Thaler, R. H., \& Benartzi, S. (2004). Save more tomorrow: Using behavioral economics to increase employee saving. Journal of Political Economy, 112, 164-187.

Viscusi, W. K., Huber, J., \& Bell, J. (2008). Estimating discount rates for environmental quality from utility-based choice experiments. Journal of Risk and Uncertainty, 37, 199-220.

Wakker, P. P., \& Deneffe, D. (1996). Eliciting von Neumann-Morgenstern utilities when probabilities are distorted or unknown. Management Science, 42, 1131-1150. 